\title{
Short communication: Recombinant bacteriophage endolysin PlyC is nontoxic and does not alter blood neutrophil oxidative response in lactating dairy cows
}

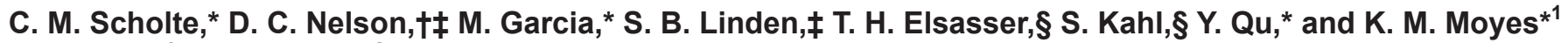 \\ *Department of Animal and Avian Sciences, and \\ †Department of Veterinary Medicine, University of Maryland, College Park 20742 \\ fInstitute for Bioscience and Biotechnology Research, University of Maryland, Rockville 20850 \\ §Agricultural Research Service, Animal Biosciences and Biotechnology, USDA, Beltsville, MD 20705
}

\section{ABSTRACT}

Mastitis is the leading cause of antimicrobial use on dairy farms. The potential for antimicrobial resistance has led to the examination of alternative strategies for controlling mastitis. One such alternative is $\mathrm{PlyC}$, a potent peptidoglycan hydrolase derived from the streptococcal $\mathrm{C} 1$ bacteriophage that causes targeted lysis of the cell wall of Streptococcus uberis. At a concentration of $1.0 \mu \mathrm{g} / \mathrm{mL}$, recombinant PlyC can induce lytic activity, suggesting that a low dose may successfully eliminate infection. We evaluated the dose effect of PlyC $(1-50 \mu \mathrm{g} / \mathrm{mL})$ on cytotoxicity and oxidative response on bovine blood polymorphonuclear leukocytes (PMN) obtained from 12 healthy, mid-lactation primiparous dairy cows. Following incubation at 0.5 and 2 $\mathrm{h}$, cytotoxicity was characterized by measuring lactate dehydrogenase release from isolated cells. Oxidative burst response was characterized as the intensity of chemiluminescence produced in the interaction of reactive oxygen species generated in response to 0 or 1.6 $\mu \mathrm{g} / \mathrm{mL}$ of phorbol 12-myristate-13-acetate (PMA) with a luminescent substrate with and without addition to $\mathrm{PlyC}$ to the incubation matrix. Data were analyzed as a complete randomized block design using mixed model procedures. Cytotoxicity of $\mathrm{PlyC}$ was not affected by concentrations up to $50 \mu \mathrm{g} / \mathrm{mL}$. As expected, PlyC cytotoxicity on PMN varied across incubation time with greater cell toxicity measured at $2 \mathrm{~h}$ of incubation as compared with $0.5 \mathrm{~h}$ and is primarily attributed to the short life of PMN ex vivo. Concentrations of PlyC up to $50 \mu \mathrm{g} / \mathrm{mL}$ did not affect oxidative response; however, oxidative response was affected by incubation time and PMA concentration. In summary, varying doses

\footnotetext{
Received September 27, 2017.

Accepted February 13, 2018.

${ }^{1}$ Corresponding author: kmoyes@umd.edu
}

of PlyC are nontoxic as estimated by lactate dehydrogenase release from cells and do not appear to alter PMA-stimulated reactive oxygen species production in bovine PMN. These early observations support continued work on the potential for application of this novel agent in combating mastitis.

Key words: mastitis, antibiotic alternative

\section{Short Communication}

With regard to dairy production, mastitis is the most common and economically significant disease. The estimated losses to producers in the United States exceed $\$ 2$ billion per year with each clinical case costing approximately $\$ 179$ (Cha et al., 2011). A wide variety of pathogens cause bovine mastitis including bacteria, yeast, and algae. Pathogens arising from the environment, such as Streptococcus uberis and Escherichia coli, are consistently being detected and reported as a leading cause of subclinical and clinical bovine mastitis throughout the world (National Mastitis Council, 2015). Streptococcus uberis is the most prevalent environmental streptococci mastitis pathogen (Jayarao et al., 1999). The number of cases caused by S. uberis continues to rise even in well-managed herds as typical mastitis control practices, such as teat disinfection and sanitary housing and milking environments, are less effective at preventing $S$. uberis-associated mastitis (Kromker et al., 2014).

The most common treatment strategy for clinical cases of $S$. uberis is the administration of intramammary antibiotics, such as cephalosporin $\beta$-lactams or penicillin G (Oliver et al., 2011). Growing concerns regarding antibiotics and the potential for antimicrobial resistance from their use have led to the development of alternative strategies for controlling mastitis (Bannantine et al., 2013). An antimicrobial alternative currently being developed is the bacteriolytic cell wall hydrolases known as lysins (or endolysins). These 
bacteriophage-derived enzymes rapidly and specifically lyse bacteria when applied exogenously (Fischetti, 2006). One of the most potent endolysins to date is the streptococcus-specific recombinant streptococcal C1 phage lysin $(\mathbf{P l y C})$. Previous research spanning over 50 yr has shown that PlyC can rapidly lyse streptococci groups A, C, and E in addition to S. uberis and Streptococcus equi and has been shown to protect mice from streptococcal infection challenge (Krause, 1957; Nelson et al., 2001). Therefore, the use of recombinant PlyC is an appealing choice for use against select streptococcal species in bovine mastitis, such as $S$. uberis.

Following bacteria entry into the mammary gland, circulating PMN (i.e., neutrophils) are the first cells recruited and then represent the most predominant somatic cell type (Burvenich et al., 1994). The initial magnitude of the PMN response will often dictate the overall effect of mastitis on the cow, as increased recruitment of PMN and greater overall PMN activity will decrease mastitis duration and severity (Rainard and Riollet, 2003). Stimuli from mammary cells and invading pathogens activate PMN to eliminate the pathogens through the production of chemical mediators, such as reactive oxygen species (ROS), and phagocytosis (Miller et al., 1993). As an alternative to pathogenic bacteria, phorbol 12-myristate-13-acetate (PMA) is commonly used to stimulate cellular aspects of oxidative metabolism and structure normally associated with bactericidal activity in vitro (Rinaldi et al., 2007; Freitas et al., 2009).

Although intramammary administration of $\mathrm{PlyC}$ is a promising new management strategy to treat $S$. uberis mastitis, the effects of $\mathrm{PlyC}$ on the bovine immune response remain unknown. Delayed appearance or decreased immunological activity of PMN can discourage the occurrence of a bacteriological cure. Potential antibiotic alternatives should not interfere with or harm the host immune response if they are to be considered useful. The objective of this study was to evaluate any cytotoxicity effects of PlyC on bovine PMN and on the PMN oxidative response to activation. We hypothesized that various concentrations of $\mathrm{PlyC}$ would be nontoxic and not alter the inflammatory response of bovine PMN in vitro.

All procedures involving the use of live animals were approved in accordance with the regulations and guidelines set forth by the USDA-Beltsville Animal Care and Use Committee.

Twelve healthy primiparous Holstein cows were used for this study. To be eligible, cows must have had a composite milk SCC $<100,000$ cells $/ \mathrm{mL}$ and cows must not have been treated for clinical signs of mastitis or any other disease during early lactation. Eligible cows were housed in tiestalls and individually fed a standard
TMR to meet the requirement of lactating dairy cows at the USDA-Beltsville Agricultural Research Center. Free choice water was available and cows were fed ad libitum twice daily to ensure 5 to $10 \%$ refusals. All cows were milked twice daily at 0730 and $1630 \mathrm{~h}$ in a double- 8 herring bone parlor with milk records recorded monthly. Cows averaged $178 \pm 4$ DIM and 40.7 $\pm 1.3 \mathrm{~kg}$ of milk/d at the time of sampling.

Recombinant PlyC was expressed and purified as previously described (Nelson et al., 2001, 2006). Briefly, E. coli were grown at $37^{\circ} \mathrm{C}$ in baffled flasks to an optical density of 1 at $600 \mathrm{~nm}$ in LB-Miller medium (Luria broth containing $10 \mathrm{~g} / \mathrm{L}$ of sodium chloride) supplemented with $100 \mu \mathrm{g} / \mathrm{mL}$ of ampicillin. Expression of $\mathrm{PlyC}$ was induced by the addition of arabinose $(0.25 \%)$. After growth for an additional $4 \mathrm{~h}$ at $37^{\circ} \mathrm{C}$, cells were harvested, lysed by sonication, and crude phage extracts were purified by ceramic hydroxyapatite resin (Bio-Rad Laboratories Inc., Hercules, CA) to remove cellular debris. As approximately 80 to $90 \%$ pure protein was eluted by $1 M$ sodium phosphate at $\mathrm{pH} 7.2$, a second round of purification by S200 size exclusion chromatography was performed to remove endotoxin. All PlyC used in experiments was produced in a single batch.

Approximately $200 \mathrm{~mL}$ of blood was collected via jugular venipuncture from each cow into $15-\mathrm{mL}$ centrifuge tubes with conical bottoms (VWR, Radnor, PA) containing $2.5 \mathrm{~mL}$ of acid-citrate dextrose solution (Sigma-Aldrich, St. Louis, MO) and stored on ice. Blood samples were processed within $1 \mathrm{~h}$ of collection. Isolation of PMN, viability, and cell differentiation were performed using endotoxin-free materials and reagents as previously described (Garcia et al., 2015). Viability of PMN was $96 \%$ with a cell differential of $91 \%$ PMN. Cells were counted by a TC20 Automated Cell Counter (Bio-Rad Laboratories) then diluted to concentrations appropriate for each assay. From each cow, $800 \mu \mathrm{L}$ of PMN were placed in 24-well cell culture plates and treated with $200 \mu \mathrm{L}$ of $0,1,10$, or $50 \mu \mathrm{g}$ of PlyC in $1 \times$ phosphate buffer solution. Plates were incubated for 0.5 or $2 \mathrm{~h}$ at $37^{\circ} \mathrm{C}, 95 \%$ relative humidity, and $5 \% \mathrm{CO}_{2}$.

The cytotoxicity of PlyC on PMN was determined in vitro. Prior to incubation with PlyC, PMN were diluted to $\sim 3.5 \times 10^{5}$ per $\mathrm{mL}$ for analysis of PMN cytotoxicity using the CytoTox 96 nonradioactive, colorimetric assay (Promega, Madison, WI) to quantify lactate dehydrogenase production. Relative cytotoxicity was calculated per instructions provided by the manufacturer (experimental divided by maximum lysis). Within incubation period and cow, each $\mathrm{PlyC}$ treatment was performed in triplicate $(\mathrm{CV}=2.66 \%)$.

The oxidative response of PMN was determined by luminol chemiluminesence assay to quantify ROS pro- 
duction. Neutrophils were diluted to $\sim 3.0 \times 10^{6}$ per $\mathrm{mL}$ before incubation with PlyC. Following incubation with $\mathrm{PlyC}$ for 0.5 or $2 \mathrm{~h}, 0.5 \mathrm{~mL}$ of medium was transferred into round-bottomed, glass 7-mL tubes. Tubes were placed into a heating block and incubated at $37^{\circ} \mathrm{C}$ for $10 \mathrm{~min}$. In a separate reaction tube $(2$ per each medium tube), $900 \mu \mathrm{L}$ of assay buffer $\left(0.5 \mathrm{mM} \mathrm{CaCl}_{2}\right.$ in Hanks' balanced salt solution) and $100 \mu \mathrm{L}$ of a $5 \mathrm{mM}$ luminol solution in dimethyl sulfoxide (Sigma-Aldrich) were added and placed in the heating block. At the end of the incubation, either $40 \mu \mathrm{L}$ of sterile water or 1.6 $\mu \mathrm{g} / \mathrm{mL}$ of PMA (Sigma-Aldrich) solution was added to each medium tube. Tubes were gently vortexed and returned to the heating block. After heating, $40 \mu \mathrm{L}$ from the medium tube was transferred to the reaction tube. Tubes were again lightly vortexed and immediately placed in the luminometer (AutoLumat LB 953, Berthold Technologies, Germany) to measure relative chemiluminescence for $20 \mathrm{~min}$. Within incubation period, PMA concentration, and cow, each PlyC treatment was performed in duplicate $(\mathrm{CV}=11.96 \%)$.

Experimental units and the corresponding sample size of cows that were used in this study have been determined using the POWER procedure of SAS (SAS/ STAT version 9.3, SAS Institute Inc., Cary, NC) for a 1-way ANOVA. Means and average standard deviation values for PMN oxidative response and PlyC cytotoxicity were calculated from a previously conducted pilot study. The following are parameters included in the procedure: (1) an $\alpha$ set at 0.05 to declare significance, (2) an $80 \%$ probability of detecting a difference in treatment means, (3) a 15,000 luminescence unit difference between treatment means, and (4) an average standard deviation value of 6,400 . The procedure was repeated for PlyC cytotoxicity with a 15 unit difference between means and an average standard deviation value of 7 . Based on the results of the power analyses, it was concluded that at least 10 cows are required for this study.

Response variables were analyzed as a complete randomized block design using the PROC MIXED procedure of SAS. Sources of variation within the model included effects of PlyC concentration, PMA concentration (oxidative response model only), and incubation time as well as 2- and 3-way interaction among the main effects. Cow was designated as a random effect in the model. Separation of least squares means for significant effects was accomplished using the PDIFF option in the MIXED procedure. Significance was declared at $P \leq 0.05$. Data are presented as least squares means \pm standard errors of the mean.

In the current study, concentrations of purified PlyC at 1,10 , and $50 \mu \mathrm{g} / \mathrm{mL}$ did not have a cytotoxic effect on PMN relative to cells incubated in buffered solution $(P=0.41)$ as cytotoxicity remained constant across

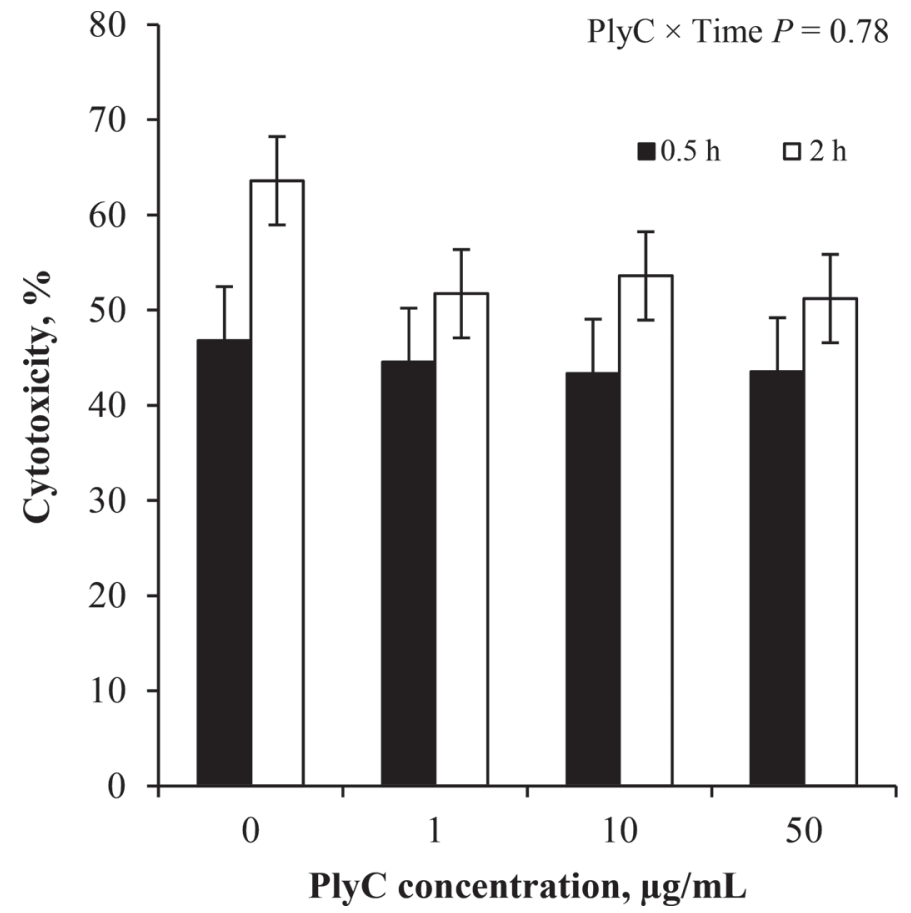

Figure 1. Least squares means and SE for bovine neutrophil cytotoxicity of neutrophils incubated in vitro for 0.5 and $2 \mathrm{~h}$ in various concentrations of recombinant streptococcal C1 phage lysin (PlyC).

concentrations (47-55\%). These results indicate that $\mathrm{PlyC}$, even at high doses, is not toxic to bovine PMN in vitro. Incubation time increased PlyC cytotoxicity by $25 \%$ from 0.5 to 2 h ( 44.6 vs. $55.0 \pm 2.8 \% ; P=0.01)$ as expected, given that the relatively short half-life of circulating PMN is 6 to $8 \mathrm{~h}$ (Summers et al., 2010). However, incubation time across PlyC concentrations did not affect cytotoxicity $(P=0.78$; Figure 1$)$.

Respiratory oxidative burst activity of PMN incubated with purified $\mathrm{PlyC}$ was determined by luminol chemiluminescence following 0.5 or $2 \mathrm{~h}$ of incubation and stimulation by 0 or $1.6 \mu \mathrm{g} / \mathrm{mL}$ of PMA. Similar to cytotoxicity, respiratory burst was not affected by PlyC concentrations up to $50 \mu \mathrm{g} / \mathrm{mL}(P=0.52)$ and relative luminescence remained constant $(29,838 \pm 3,315$ relative luminescence units), indicating that $\mathrm{PlyC}$ may not interfere with the function of PMN during the inflammatory response in vivo. As expected, increasing PMA concentration from 0 to $1.6 \mu \mathrm{g} / \mathrm{mL}$ increased PMN respiratory burst response $(21,514$ vs. $38,162 \pm 4,585$ relative luminescence units; $P<0.01)$ reflecting the activation of NADPH oxidase and production of ROS, superoxide anions, and hydrogen peroxide, similarly to that of an invading pathogen (White et al., 1984; Kobayashi et al., 2005). However, PMN respiratory response to PMA was dependent upon incubation time with longer incubation decreasing respiratory burst of 

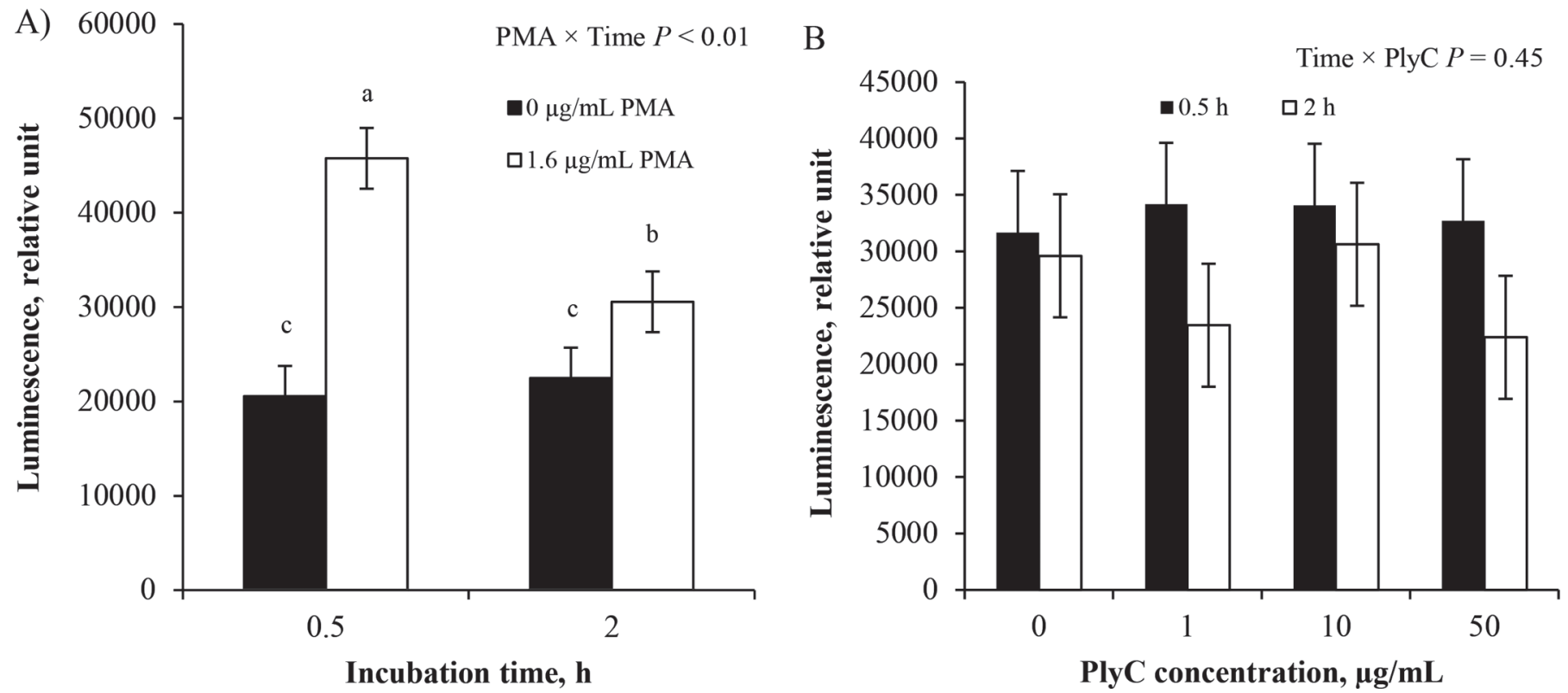

Figure 2. Least squares means and SE for the bovine neutrophil oxidative response as determined by luminol chemiluminescence at 0.5 or 2 $\mathrm{h}$ of incubation and stimulated by 0 or $1.6 \mu \mathrm{g} / \mathrm{mL}$ of phorbol 12 -myristate-13-acetate (PMA) solution in various concentrations of recombinant streptococcal $\mathrm{C} 1$ phage lysin (PlyC). (A) Oxidative response and incubation time across PMA concentration; (B) oxidative response and incubation time across PlyC concentration. Means with different letters $(\mathrm{a}-\mathrm{c})$ differ $(P<0.05)$.

PMN incubated with $1.6 \mu \mathrm{g} / \mathrm{mL}$ of PMA $(P<0.01$; Figure 2A). The overall decreased respiratory burst response across incubation time reflects the increased cell deterioration and death observed in the PMN cytotoxicity analysis. The relative oxidative burst of PMN was not affected by $\mathrm{PlyC}$ concentration across incubation time $(P=0.45)$, although relative luminescence was numerically lower following $2 \mathrm{~h}$ incubation (Figure $2 \mathrm{~B}$ ).

The growing concern for antibiotic resistance and improved stewardship has fueled the drive to find antibiotic and antimicrobial alternatives. Bacteriophage lysins have been used successfully in a variety of models to control pathogenic antibiotic resistant and tolerant bacteria found on mucosal surfaces and infected tissues. The streptococcal-specific endolysin $\mathrm{PlyC}$ is unique among the lysins and has rapid lethal effect in vitro (McGowan et al., 2012) and in vivo (Nelson et al., 2001). Relatively low concentrations of PlyC have successfully been used for disinfection of $S$. equi $(1 \mu \mathrm{g} / \mathrm{mL}$; Hoopes et al., 2009) and degradation of Streptococcus pyogenes biofilms $(5 \mu \mathrm{g} / \mathrm{mL}$; Shen et al., 2013) in addition to the prevention and elimination of $S$. pyogenes in mice (0.01 $\mu \mathrm{g}$; Nelson et al., 2001). These effective concentrations were substantially lower than those examined in the current study. Concentrations of PlyC up to 50 $\mu \mathrm{g} / \mathrm{mL}$ were nontoxic on bovine PMN. These results demonstrate that the lytic activity of PlyC does not target bovine PMN and that effective concentrations of
PlyC would likely be nontoxic to recruited neutrophils during mastitis.

Recruited neutrophils use many techniques to destroy pathogenic bacteria that have entered the mammary gland, such as phagocytosis, extracellular traps, and degranulation (Summers et al., 2010). Reactive oxidative species produced and released during the respiratory burst response aid in bacterial destruction and clearance of the infection. Again, high concentrations of PlyC (up to $50 \mu \mathrm{g} / \mathrm{mL}$ ) did not affect the respiratory burst response of bovine PMN. Effective lower concentrations of $\mathrm{PlyC}$ would likely not affect the respiratory response of PMN during the immunological response to infection.

To our knowledge, this study is the first to characterize the effects of bacteriophage-based lytic enzyme PlyC on bovine PMN. Results show that high concentrations of $\mathrm{PlyC}$ are nontoxic and do not impede the oxidative response of bovine PMN. Future studies investigating the use of $\mathrm{PlyC}$ as an alternative therapy for treatment of mastitis are warranted.

\section{ACKNOWLEDGMENTS}

This study was partly funded via the Maryland Agricultural Experiment Station and USDA-NIFA (Proposal \#2015-06929). The authors acknowledge the staff of the University of Maryland Dairy Research Unit 
(Clarksville, MD) for their assistance with animal care and data collection.

\section{REFERENCES}

Bannantine, J. P., S. C. Olsen, M. E. Kehrli Jr., T. B. Stanton, D. L. Casas, D. L. Whipple, and K. A. Zuekle. 2013. High-impact animal health research conducted at the USDA's National Animal Disease Center. Vet. Microbiol. 165:224-233.

Burvenich, C., M. J. Paape, A. W. Hill, A. J. Guidry, R. H. Miller, R. Heyneman, W. D. J. Kremer, and A. Brand. 1994. Role of the neutrophil leukocyte in the local and systemic reactions during experimentally induced $E$. coli mastitis in cows immediately after calving. Vet. Q. 16:45-50.

Cha, E., D. Bar, J. A. Jerti, L. W. Tauer, G. Bennett, R. N. Gonzalez, Y. H. Schukken, F. L. Welcome, and Y. T. Grohn. 2011. The cost and management of different types of clinical mastitis in dairy cows estimated by dynamic programming. J. Dairy Sci. 94:4476-4487.

Fischetti, V. A. 2006. Using phage lytic enzymes to control pathogenic bacteria. BMC Oral Health 6(Suppl 1):S16.

Freitas, M., G. Porto, J. L. Lima, and E. Fernandes. 2009. Optimization of experimental settings for the analysis of human neutrophils oxidative burst in vitro. Talanta 78:1476-1483.

Garcia, M., T. H. Elsasser, D. Biswas, and K. M. Moyes. 2015. The effect of citrus-derived oil on bovine blood neutrophil function and gene expression in vitro. J. Dairy Sci. 98:918-926.

Hoopes, J. T., C. J. Stark, H. A. Kim, D. J. Sussman, D. M. Donovan, and D. C. Nelson. 2009. Use of a bacteriophage lysin, PlyC, as an enzyme disinfectant against Streptococcus equi. Appl. Environ. Microbiol. 75:1388-1394.

Jayarao, B. M., B. E. Gillespie, M. J. Lewis, H. H. Dowlen, and S. P. Oliver. 1999. Epidemiology of Streptococcus uberis intramammary infections in a dairy herd. Zentralbl Veterinarmed B 46:433-442.

Kobayashi, S. D., J. M. Voyich, C. Burlak, and F. R. DeLeo. 2005. Neutrophils in the innate immune response. Arch. Immunol. Ther. Exp. (Warsz.) 53:505-517.

Krause, R. M. 1957. Studies on bacteriophages of hemolytic streptococci. I. Factors influencing the interaction of phage and susceptible host cell. J. Exp. Med. 106:365-384.
Kromker, V., F. Reinecke, J. H. Paduch, and N. Grabowski. 2014. Bovine Streptococcus uberis intramammary infections and mastitis. Clin. Microbiol. 3:157-163.

McGowan, S., A. M. Buckle, M. S. Mitchell, J. T. Hoopes, D. T. Gallagher, R. D. Heselpoth, Y. Shen, C. F. Reboul, R. H. P. Law, V. A. Fischetti, J. C. Whisstock, and D. C. Nelson. 2012. X-ray crystal structure of the streptococcal specific phage lysin PlyC. Proc. Natl. Acad. Sci. USA 109:12752-12757.

Miller, J. K., E. Brzezinska-Slebodzinska, and F. C. Madsen. 1993. Oxidative stress, antioxidants and animal function. J. Dairy Sci. $76: 2812-2823$.

National Mastitis Council. 2015. A Practical Look at Environmental Mastitis. National Mastitis Council, Madison, WI.

Nelson, D., L. Loomis, and V. A. Fischetti. 2001. Prevention and elimination of upper respiratory colonization of mice by group A streptococci by using a bacteriophage lytic enzyme. Proc. Natl. Acad. Sci. USA 98:4107-4112.

Nelson, D., R. Schuch, P. Chahales, S. Zhu, and V. A. Fischetti. 2006 PlyC: A multimeric bacteriophage lysin. Proc. Natl. Acad. Sci. USA 103:10765-10770.

Oliver, S. P., S. E. Murinda, and B. M. Jayarao. 2011. Impact of antibiotic use in adult dairy cows on antimicrobial resistance of veterinary and human pathogens: A comprehensive review. Foodborne Pathog. Dis. 8:337-355.

Rainard, P., and C. Riollet. 2003. Mobilization of neutrophils and the defense of the bovine mammary gland. Reprod. Nutr. Dev. 43:439-457.

Rinaldi, M., P. Moroni, M. J. Paape, and D. D. Bannerman. 2007. Evaluation of assays for the measurement of bovine neutrophil reactive oxygen species. Vet. Immunol. Immunopathol. 115:107-125.

Shen, Y., T. Köller, B. Kreikemeyer, and D. C. Nelson. 2013. Rapid degradation of Streptococcus pyogenes biofilms by PlyC, a bacteriophage-encoded endolysin. J. Antimicrob. Chemother. 68:18181824.

Summers, C., S. M. Rankin, A. M. Condliffe, N. Singh, A. M. Peters, and E. R. Chilvers. 2010. Neutrophil kinetics in health and disease. Trends Immunol. 31:318-324.

White, J. R., C. Huang, J. M. Hill, P. H. Naccache, E. L. Becker, and R. I. Sha'afi. 1984. Effect of phorbol 12-myristate 13-acetate and its analogue $4 \alpha$-phorbol 12,13-didecanoate on protein phosphorylation and lysosomal enzyme release in rabbit neutrophils. J. Biol. Chem. 259:8605-8611. 